\title{
Novel Technique for Inducing Neural Crest Fate in Embryonic Stem Cells
} (Stem Cells 2009;27:2896-2905)

\section{Jung Hwan $\mathrm{Oh}^{1}$ and Gunjan Tiwari ${ }^{2 *}$}

${ }^{1}$ Division of Gastroenterology, College of Medicine, The Catholic University of Korea, Seoul, Korea; and ${ }^{2}$ Division of Gastroenterology and Hepatology, Stanford University Medical Center, Stanford, CA, USA

\section{Summary}

Hotta et $\mathrm{al}^{1}$ has reported a study entitled "Small-molecule induction of neural crest-like cells derived from human neural progenitors" in the December 2009 issue of Stem Cells. Neural crest (NC) cells are ectoderm-derived multipotent migratory cells that give rise to diverse cell lineages in a developing embryo. Migratory or proliferative deficit of NC cells results in many developmental disorders. Stem cell-based therapies are potential treatment options for such disorders. The enteric nervous system (ENS) of vertebrates predominantly develops from NC cells originating from the vagal end of the neural tube. In Hirschsprung's disease, the distal bowel lacks an ENS caused by a failure of colonization by NC-derived cells. This study shows a simple method of coculture with mouse embryonic fibroblasts (mEFs) for generating NC-like cells from human embryonic stem cells (hESCs). The authors also demonstrated that subsequent exposure to Y27632, a selective inhibitor of the Rho-associated coiled kinase (ROCK), significantly increased the efficiency of differentiation into NC-like cells. These hESC-derived NC-like cells expressed NC markers including p75 (p75NTR low affinity neurotrophin receptor), SoxE (Sox8, Sox9 and
Sox10) and HNK1 (human natural killer-1 antigen). The mEFs induced NC-like cells were also able to migrate along NC pathways in avian embryos and differentiate into cells expressing neuronal and glial markers. The hESC-derived NC-like cells also demonstrated migration in mouse gut explants, however, Y27632 treatment was required for differentiation into neuronal and glial cells.

\section{Comment}

ENS is a complex neural network that controls most of the gut functions by regulating motility, secretion/absorption and vascular tone. ${ }^{2}$ The neurons and glia that comprise the ENS are derived from the migrating NC cells. ${ }^{3}$ Many developmental and acquired disorders of ENS are characterized by loss of neurons. ${ }^{4}$ One of the best-characterized enteric neuropathy is Hirschsprung's disease, which is caused by an absence of neurons in distal gastrointestinal tract. Surgical intervention is often the only treatment option for these disorders but is still plagued by postoperative morbidity. Replacement of the ENS has been considered a potential and futuristic treatment strategy. Stem cell transplantation is one such tool for replenishing the ENS. Several sources of stem cells for generating enteric neurons have been in-

Received: June 2, 2011 Revised: June 10, 2011 Accepted: June 12, 2011

(c) This is an Open Access article distributed under the terms of the Creative Commons Attribution Non-Commercial License (http://creativecommons org/licenses/by-nc/3.0) which permits unrestricted non-commercial use, distribution, and reproduction in any medium, provided the original work is properly cited.

*Correspondence: Gunjan Tiwari, MD

Division of Gastroenterology and Hepatology, Stanford University Medical Center, 300 Pasteur drive, Stanford, CA 94305, USA

Financial support: None.

Tel: +1-650-498-7858, Fax: +1-650-723-5488, E-mail: gtiwari@stanford.edu

Conflicts of interest: None. 
vestigated, including CNS derived neural stem cells, enteric neural progenitors and NC cells isolated from PNS or neural tube., This paper demonstrated that embryonic stem (ES) cells can also be a potential source of enteric neurons by inducing development of NC-like cells upon coculture with $\mathrm{mEFs}$ and treatment with small molecule inhibitor of ROCKI/II. ES cells-derived NC-like cells can therefore potentially become an unlimited source of stem cells for enteric neuropathies.

ES cells have previously been shown to spontaneously give rise to $\mathrm{NC}$-like cells or their derivatives in culture while exposure to stromal cells increased the efficiency. Hotta et $\mathrm{al}^{1}$ demonstrated that coculture with $\mathrm{mEFs}$ could also increase the efficiency of developing NC-like cells from ES cells, which was comparable to stromal cell exposure. The mEF-induced $\mathrm{NC}$ cells express the $\mathrm{NC}$ specific markers, and they migrate and differentiate in vitro in avian embryos and mouse gut explants. Furthermore, the authors demonstrated that Y27632 treatment increased the proportion of migrating NC-like cells derived from hESC in vitro. Y27632 is a ROCK I/II inhibitor, which selectively targets p160ROCK from the family of Rho-associated protein kinases. ${ }^{7}$ Inhibition of Rho/ROCK signaling has been implicated in the epithelial-mesenchymal transition of NC cells from avian neural epithelium. ${ }^{8}$ Epithelial-mesenchymal transition of neuroepithelial cells is the key step for neural tube formation and induction of the NC. The authors demonstrated that the hESC-derived NC-like cells behaved similarly to NC cells in avian embryos in that they assembled along NC migratory pathways and differentiated into neuronal $(\mathrm{Hu}$, the generic neuronal marker positive) and glial cells (S100b positive) within the embryonic peripheral nervous system. This study also reports the integration of hESC-derived cells into the developing gut for the first time. However, further studies investigating the functional properties of the hESC-derived neurons are required before successful translation of this technique.

Post transplant survival of cells in any cell-based therapy remains a big challenge and represents a critical limiting factor in successful repopulation. Tissue derived NC cells can be gen- erated autologously and therefore cannot be immunerejected post transplantation leading to higher success rate. The authors discussed the potential of immunerejection of hESC-derived NC cells in transplantations since ESCs were not patient specific. They, however, suggested that patient specific induced pluripotent stem cells (iPSCs) might be able to fill this gap.' However, successful transfer of methodology developed in hESCs to iPSCs remains to be seen.

In conclusion, this study describes a simple and efficient method for generating NC-like cells from hESCs. These NC-like cells can potentially be a superior and unlimited source of cells for cell-based therapies of enteric neuropathies.

\section{References}

1. Hotta R, Pepdjonovic L, Anderson RB, et al. Small-molecule induction of neural crest-like cells derived from human neural progenitors. Stem Cells 2009;27:2896-2905.

2. Wood JD, Alpers DH, Andrews PL. Fundamentals of neurogastroenterology. Gut 1999;45(suppl 2):II6-II16.

3. Newgreen D, Young HM. Enteric nervous system: development and developmental disturbances - part 1. Pediatr Dev Pathol 2002;5: 224-247.

4. De Giorgio R, Camilleri M. Human enteric neuropathies: morphology and molecular pathology. Neurogastroenterol Motil 2004;16: 515-531.

5. Micci MA, Pasricha PJ. Neural stem cells for the treatment of disorders of the enteric nervous system: strategies and challenges. Dev Dyn 2007;236:33-43.

6. Metzger M, Caldwell C, Barlow AJ, Burns AJ, Thapar N. Enteric nervous system stem cells derived from human gut mucosa for the treatment of aganglionic gut disorders. Gastroenterology 2009;136: 2214-2225.e1-e3.

7. Uehata M, Ishizaki $T$, Satoh $H$, et al. Calcium sensitization of smooth muscle mediated by a Rho-associated protein kinase in hypertension. Nature 1997;389:990-994.

8. Groysman M, Shoval I, Kalcheim C. A negative modulatory role for rho and rho-associated kinase signaling in delamination of neural crest cells. Neural Dev 2008;3:27-48.

9. Nishikawa $S$, Goldstein RA, Nierras CR. The promise of human induced pluripotent stem cells for research and therapy. Nat Rev Mol Cell Biol 2008;9:725-729. 